\title{
Safer Conception and Family Planning Knowledge, Attitudes, and Practices Among Postpartum Women with HIV in Rural South Africa
}

This article was published in the following Dove Press journal: Open Access Journal of Contraception

\author{
Lissa N Mandell $\mathbb{B}^{\prime}$ \\ Violeta J Rodriguez (iD) ${ }^{1,2}$ \\ Deborah L Jones (iD) \\ 'Department of Psychiatry and \\ Behavioral Sciences, University of Miami \\ Miller School of Medicine, Miami, FL, \\ USA; ${ }^{2}$ Department of Psychology, \\ University of Georgia, Athens, GA, USA
}

Introduction: With the help of safer conception strategies (SCS), women with HIV (WHIV) can achieve their reproductive goals while minimizing the risk of transmission to their partners or infants. However, interpregnancy intervals of at least 24 months are recommended to optimize maternal and infant health outcomes, so postpartum WHIV need to use contraception to delay subsequent pregnancies. Understanding safer conception and family planning knowledge, attitudes, and practices among WHIV is key to tailoring family planning policy and intervention development in regions with high HIV prevalence.

Methods: This study described the safer conception and family planning knowledge, attitudes, and practices of postpartum WHIV $(\mathrm{N}=956)$ in rural Mpumalanga province, South Africa, 12 months after delivery.

Results: Almost all women understood the importance of condom use, but most overestimated the risk of sexual transmission. A majority of women reported that their partner's desires (53\%) and the risk of perinatal transmission (58\%) were very important factors when making childbearing decisions. Most women (81\%) used condoms for HIV prevention and most $(83 \%)$ used contraception for pregnancy prevention. Many women (33\% of contraceptive users) used condoms for both HIV prevention and contraception without using another contraceptive method as well. Only $43 \%$ of contraceptive users endorsed dual method use of condoms with hormones, intrauterine devices, or sterilization.

Discussion: Results highlight the prominence of condom-based pregnancy and HIV transmission prevention, the influence of male partners in fertility decision-making, and the opportunity for further education and promotion of long acting methods in this setting.

Keywords: HIV, family planning, contraception, condoms, pregnancy, safer conception

\section{Introduction}

Safer conception strategies (SCS) for HIV-affected couples, such as pre-exposure prophylaxis (PrEP) for HIV prevention in serodiscordant couples and antiretroviral therapy (ART) treatment as prevention (TasP), as well as timed unprotected intercourse, have made it possible for women to conceive with significantly less risk of HIV infection or transmission. ${ }^{1-3}$ When combined with strategies for prevention of mother-to-child transmission (PMTCT), women with HIV (WHIV) can achieve their reproductive goals while protecting their partners and infants from HIV infection. Due to greatly reduced risk of HIV transmission when using SCS and PMTCT strategies, along with high fertility intentions and HIV burden among women of reproductive age, a reproductive rights approach toward managing the
Correspondence: Deborah L Jones Email d.jones3@med.miami.edu
Open Access Journal of Contraception 2021:12 17-25 
reproductive health of people with HIV (PHIV) has been promoted by several advocacy organizations and countries, including South Africa. ${ }^{4,5}$ South Africa has adopted national guidelines for healthcare providers and facilities to help PHIV and their partners achieve their reproductive goals, ${ }^{6}$ but these family planning discussions including SCS are not consistently couples-based, implemented, or understood $^{5}$ and some ART methods are not available for safer conception in resource-limited settings. ${ }^{7}$ Understanding the knowledge, attitudes, and practices of PHIV regarding safer conception, family planning, contraception, and fertility decision-making is needed to inform services designed to increase uptake of safer conception and contraception methods.

One of the primary motivators for childbearing in South Africa is the societal expectation of women, especially married women, to have children. ${ }^{8-12}$ Bearing children is viewed as a woman's responsibility and critical to fulfilling female gender roles. ${ }^{8,11,12}$ However, in spite of cultural expectations to bear children, childbearing by PHIV may be stigmatized in the community. ${ }^{11,12}$ PHIV also express concern about personal health issues, orphaning a child, HIV disclosure, and infecting their child or their partner. ${ }^{8,9,11}$

In addition to expectations and concerns regarding childbearing, child spacing (increasing the length of time between pregnancies) is encouraged. The World Health Organization (WHO) recommends waiting at least 24-36 months after childbirth before becoming pregnant again to reduce the risk of adverse maternal and child health outcomes; ${ }^{13}$ interpregnancy intervals of more than 36 months may be even more advantageous. ${ }^{14}$ In fact, the perinatal period is associated with greater risk of HIV acquisition due to both physiological and behavioral factors. ${ }^{15}$ As such, fertility planning discussions between healthcare providers and postpartum women can play a key role in addressing the role of effective contraceptive and prevention methods to prevent pregnancy and HIV subsequent to giving birth.

Dual method use - the use of both condoms and another highly effective contraceptive method ${ }^{16}$ - is therefore recommended to protect against both HIV and pregnancy. In addition, ART adherence is essential even if both partners are living with HIV, due to the risk of HIV superinfection (becoming infected with a different strain of HIV) or infection with drug-resistant virus. ${ }^{17}$ When WHIV want to conceive, the use of safer conception strategies is critical to reducing the risk of transmission to her partner. ${ }^{1}$ However, existing research in South Africa has found low knowledge of safer conception strategies among HIV-affected individuals. ${ }^{11,18-20}$

Rural areas of South Africa are particularly hard-hit by HIV and have high HIV prevalence rates. ${ }^{21}$ Rural clinics also face resource and personnel limitations, and patients in over-extended antenatal clinics may have less access to reproductive health services. ${ }^{22,23}$ In addition, although South Africa is a male-dominated society in general, this may be especially true in rural areas, where male partners are more likely to dominate reproductive decisionmaking $^{24}$ and women may feel even more pressure to prove that they are fertile. ${ }^{23}$

To optimize reproductive health policy uptake, enhance contraceptive use, prevent HIV transmission, help families achieve reproductive goals, and optimize maternal and child health in rural areas, safer conception and family planning knowledge, attitudes, and practices among postpartum WHIV in rural Mpumalanga province, South Africa were examined. It was anticipated that information gained could be used to inform service provision in rural South Africa to better reach vulnerable populations in the region.

\section{Materials and Methods}

The results presented here are drawn from data from a two-phase, longitudinal, cluster-randomized clinical trial of "Protect Your Family" (PYF), a cognitivebehavioral PMTCT intervention delivered by lay healthcare workers, conducted in rural Mpumalanga province, South Africa. ${ }^{25}$ The trial used a $2 \times 2$ factorial design: two phases (Phase 1 and Phase 2) and two conditions (intervention and control). Prior to study procedures, ethical approval was obtained from the associated South African Research Ethics Committee, the associated US Institutional Review Board, and the Mpumalanga provincial government. The study was conducted in accordance with the Declaration of Helsinki. All participants provided written informed consent prior to participation.

\section{Participants}

Participants were postpartum women with HIV in rural Mpumalanga recruited from 12 different community health centers (CHCs) with antenatal clinical services. Candidates were recruited during pregnancy to participate in the PYF clinical trial. Participants were at least 18 years old, had been diagnosed as HIV-seropositive, were between 6 and 30 weeks pregnant, and had a male partner. 
Women completed study assessments twice prenatally and three times postnatally. The results presented here use data from the 12-months postpartum assessment collected from June 2015 through April 2018.

All participants received the standard of care Department of Health antenatal PMTCT education sessions, which cover topics such as partner testing, infant feeding, safer sex, ART adherence, family planning, and infant prophylaxis. ${ }^{26}$ Intervention participants also attended PYF education sessions, which used cognitive-behavioral strategies to further address these topics, as well as family relationships and partner communication. Control participants also watched video presentations about health issues such as fevers and burns. Women in Phase 2 participated in the study with their male partners; women in Phase 1 were required to have a male partner, but the partner did not participate in the study. Further information can be found in the published study protocol. ${ }^{25}$

\section{Assessments}

Assessments and intervention/control sessions were conducted at the CHCs. To reduce desirability bias and accommodate all literacy levels, participants completed questionnaires using an audio computer-assisted selfinterview (ACASI) system in their preferred language (English, isiZulu, or Sesotho).

\section{Measures}

\section{Demographics and HIV History}

Demographic and HIV history variables assessed included age, relationship status, number of children (prior to the index pregnancy), monthly household income, educational attainment, perceived partner HIV status, disclosure of HIV status to partner by 12-months postpartum, and whether women were diagnosed with HIV during the index pregnancy. These demographic and HIV history variables were assessed at baseline, except for disclosure of HIV status to partner by 12-months postpartum.

\section{Knowledge}

\section{Knowledge of safer conception strategies.}

SCS knowledge was assessed using eight items adapted from the Safer Conception Knowledge, Attitudes, and Practices (SCKAP) and Family Planning Survey. ${ }^{27}$ The questions assessed knowledge of safer conception methods, horizontal transmission risk, and timing of the fertile window. Each response was scored as correct (1 point) or incorrect ( 0 points), for a maximum total score of 8 points.

\section{Attitudes}

Fertility intentions and reproductive decision-making.

Fertility intentions were assessed with the question "Are you planning to have more children in the future?" to which participants responded with "yes" or "no". Participants were asked to rate the importance of four different factors impacting fertility decision-making, using the prompt "When you make the decision whether or not to have a baby, how important are the following things?" The four factors were "your family's desire for you to have a baby (or not)", "your partner's desire for you to have a baby (or not)", "other people's opinion about whether you should have a baby (or not)", and "the possibility for your baby to become infected with HIV". Each item used a scale ranging from 1 ("not important at all") to 10 ("very important").

\section{Practices}

Pregnancy planning, intentions, and contraception.

Participants were asked "Are you currently pregnant?" and responded with "Yes", "No", or "I do not know". Those indicating "yes" were also asked "Was this a planned pregnancy? (Yes/No)". Participants were asked "Not including your study visits, have you talked with a health care provider about trying to get pregnant in the future? (Yes/No)". Condom use for HIV prevention was assessed with the question "Are you currently using condoms to prevent HIV transmission? (Yes/No)". Participants were asked "Are you and your partner currently using contraception to prevent pregnancy? (Yes/ No)". Those who said "yes" were then asked which methods they were using and could select more than one method. The methods listed were condoms, oral contraceptive pills, injections, intrauterine device (IUD), implant, tubal ligation, vasectomy, early withdrawal, lactation amenorrhea, rhythm/calendar method, diaphragm, and spermicide. Women could also choose "do not know" or "one of us is infertile". Women who used contraceptives were also categorized as long-term method users or short-term method users. Long-term method users were those who indicated that they used at least one long-term method (IUD, implant, tubal ligation, vasectomy, or infertility). Short-term method users were those who used contraceptives (and answered the questions about methods) but did not use any long-term methods. Women who indicated that they were pregnant at the time of the assessment were not asked about contraception. 
Dual method use.

Participants (excluding those who were pregnant at the time of the assessment) were classified as dual method users or non-dual method users. Dual method use was defined as using condoms to prevent HIV transmission and also using at least one highly effective contraceptive method to prevent pregnancy. "Highly effective" methods included oral contraceptive pill, injection, implant, IUD, tubal ligation, vasectomy, or infertility. Lactation amenorrhea was not classified as highly effective as it is only considered "effective" for 6 months after childbirth. ${ }^{28}$

\section{Results}

\section{Sociodemographic Characteristics of Women}

The sample consisted of $\mathrm{N}=956$ women; however, $\mathrm{n}=13$ did not have demographic data due to technical difficulties. Women $(\mathrm{n}=943)$ were, on average, $28.54(\mathrm{SD}=$ 5.82 ) years old. About half (53\%) of women were diagnosed with HIV during the current pregnancy. Slightly more than half $(55 \%)$ were unmarried and not living with their partner, 26\% were unmarried living together with their partner, and 19\% were married. Approximately one-third $(30 \%)$ of women reported that their partner was HIV-seropositive; the majority of women (82\%) reported they had disclosed their HIV status to their partner. Most women $(80 \%)$ had at least one child before the most recent pregnancy, and $67 \%$ had a monthly household income of 312 South African Rand (USD\$18 as of the time of this writing) or more. One-third (32\%) of women had completed 12 years of education or more.

\section{Knowledge}

On average, women had a total score of $M=3.88$ (SD = 1.03 ) on the safer conception knowledge scale (maximum score possible: 8 ). Correct answers for all items in the scale are presented in Table 1. Overall, most women understood that condom use was important, but were less knowledgeable about safer conception methods. A majority of women overestimated the risk of partner infection; 617 women (65\%) believed there was a $100 \%$ chance of male partner infection when trying to get pregnant. A sizeable portion $(n=185 ; 19 \%)$ believed there was no risk of partner infection when trying to get pregnant.

\section{Attitudes}

Nearly one-third $(30 \%)$ of women reported planning to have more children in the future. The most important influences on childbearing decisions were (in order of importance) infants, partners, family members, and other people. More than half $(58 \%)$ of women considered the possibility of their infant becoming infected to be very important. About one-third (36\%) of women reported that their family's desire for her to have a child (or not) was very important; half (53\%) of reported that their partner's desire to have a child (or not) was very important; a third (30\%) considered other people's opinion to be very important. Attitudes are summarized in Table 2.

\section{Practices}

Most (81\%) women, regardless of pregnancy status, were using condoms to prevent HIV transmission. Within twelve months of their last pregnancy, 4\% $(n=40)$ of

Table I Knowledge $(\mathrm{N}=956)$

\begin{tabular}{|c|c|}
\hline Item & $\begin{array}{l}\mathbf{N}(\%) \\
\text { Correct }\end{array}$ \\
\hline I. When trying to get pregnant, how much is a man at risk of HIV infection if the woman is positive? ${ }^{\mathrm{a}}$ & $154(16.1 \%)$ \\
\hline $\begin{array}{l}\text { 2. When trying to get pregnant, what are the chances that the man would be infected with HIV if the woman is taking HIV } \\
\text { medications? }\end{array}$ & $377(39.4 \%)$ \\
\hline 3. When trying to get pregnant, when in her monthly cycle is a woman the most likely to become pregnant? ${ }^{c}$ & $182(19.0 \%)$ \\
\hline 4. When trying to get pregnant, how can a woman minimize the risk of her partner getting HIV if she is HIV positive? & \\
\hline 4a. By only having sexual intercourse without a condom when she is the most fertile? ${ }^{d}$ & $556(58.1 \%)$ \\
\hline 4b. By taking her HIV medications? ${ }^{\mathrm{d}}$ & $531(55.5 \%)$ \\
\hline 5. When a woman is pregnant, does a man need to use a condom during her pregnancy if she is HIV positive? ${ }^{d}$ & $852(89.1 \%)$ \\
\hline 6. After a woman delivers her baby, does a man need to use a condom if she is HIV positive? ${ }^{d}$ & $881(92.2 \%)$ \\
\hline 7. If a woman is breastfeeding, does a man need to use a condom if she is HIV positive? ${ }^{d}$ & $885(92.6 \%)$ \\
\hline
\end{tabular}

Notes: ${ }^{a}$ Options ranged from I (No Chance of Infection) to 9 ( $100 \%$ will be infected); non-absolutes (answers between 2 and 8 ) were considered correct. ${ }^{b}$ Options ranged from I (No Chance of Infection) to 9 ( $100 \%$ will be infected); values $<9$ were considered correct. ${ }^{\circ}$ Options were a) During her bleeding, b) The weeks in the middle of the cycle (correct answer), c) The week after the bleeding, d) The week before the bleeding, e) I do not know. 'Options were a) No, b) Yes (correct), and c) I do not know. 
Table 2 Attitudes $(\mathrm{N}=956)$

\begin{tabular}{|c|c|}
\hline Item & $\mathbf{N}(\%)$ \\
\hline Planning to have more children in the future & $284(29.7 \%)$ \\
\hline \multicolumn{2}{|c|}{$\begin{array}{l}\text { When you make the decision whether or not to have a baby, } \\
\text { how important are the following things? }\end{array}$} \\
\hline Your family's desire for you to have a baby (or not)? & \\
\hline I - Not important at all & $508(53.1 \%)$ \\
\hline $2-9$ & $101(10.6 \%)$ \\
\hline 10 - Very important & $347(36.3 \%)$ \\
\hline Your partner's desire for you to have a baby (or not)? & \\
\hline I - Not important at all & $330(34.5 \%)$ \\
\hline $2-9$ & $124(13.0 \%)$ \\
\hline 10 - Very important & $502(52.5 \%)$ \\
\hline $\begin{array}{l}\text { Other people's opinion about whether you should } \\
\text { have a baby (or not)? }\end{array}$ & \\
\hline I - Not important at all & $552(57.7 \%)$ \\
\hline $2-9$ & $118(12.3 \%)$ \\
\hline 10 - Very important & $286(29.9 \%)$ \\
\hline $\begin{array}{l}\text { The possibility for your baby to become infected with } \\
\text { HIV? }\end{array}$ & \\
\hline I - Not important at all & $277(29.0 \%)$ \\
\hline $2-9$ & $124(13.0 \%)$ \\
\hline 10 - Very important & $555(58.1 \%)$ \\
\hline
\end{tabular}

women were pregnant again; of these, $33 \%(n=13)$ reported that the pregnancy was planned. Most (83\%) women who were not again pregnant reported using contraception to prevent pregnancy; $41 \%$ of non-pregnant women were using highly effective contraceptive methods. About a third (35\%) of non-pregnant women were considered "dual method users" - using condoms for HIV prevention along with a highly effective contraceptive method. Among the contraceptive users who answered the question about contraceptive methods, a small proportion (5\%) used at least one long-term method (IUD, implant, tubal ligation, vasectomy, or "one of us is infertile"). Lastly, almost half (46\%) of women reported discussing future pregnancies with a healthcare provider. Practices are summarized in Table 3 and Figure 1.

\section{Discussion}

This study examined safer conception and family planning knowledge, attitudes, and practices among postpartum WHIV in rural Mpumalanga province, South Africa 12 months after delivery. Results highlight influences on fertility decision-making and demonstrate both successes and
Table 3 Practices $(\mathrm{N}=956)$

\begin{tabular}{|c|c|}
\hline Item & $\mathbf{N}(\%)$ \\
\hline Condom use to prevent HIV & $778(81.4 \%)$ \\
\hline Pregnant & 40 (4.2\%) \\
\hline Planned pregnancy $(n=40)$ & $13(32.5 \%)$ \\
\hline Using contraception to prevent pregnancy ${ }^{a}$ & $756(82.5 \%)$ \\
\hline Using highly effective contraceptive method ${ }^{\mathrm{a}, \mathrm{b}}$ & $377(4 I .2 \%)$ \\
\hline \multicolumn{2}{|l|}{ Contraceptive method $(n=678)^{c, d}$} \\
\hline Early withdrawal & $16(2.4 \%)$ \\
\hline Breastfeeding/Lactation Amenorrhea & $31(4.6 \%)$ \\
\hline Rhythm/Calendar/Natural Method & $19(2.8 \%)$ \\
\hline Condoms & $478(70.5 \%)$ \\
\hline Oral Contraceptive Pill & $59(8.7 \%)$ \\
\hline Diaphragm & $5(0.7 \%)$ \\
\hline Spermicide & $7(1.0 \%)$ \\
\hline Injection/Depo-Provera & $298(44.0 \%)$ \\
\hline Norplant (implant) & $10(1.5 \%)$ \\
\hline Intrauterine Device (IUD) & $5(0.7 \%)$ \\
\hline Tubal Ligation & $15(2.2 \%)$ \\
\hline Vasectomy & $13(1.9 \%)$ \\
\hline Infertility from either partner & $5(0.7 \%)$ \\
\hline Dual method use $\mathrm{e}^{\mathrm{a}, \mathrm{e}}$ & $323(35.3 \%)$ \\
\hline Long-term method $(n=678)^{d, f}$ & $36(5.3 \%)$ \\
\hline Discussed future pregnancies with provider & $438(45.8 \%)$ \\
\hline
\end{tabular}

Notes: ${ }^{a} n=916$, excluding $n=40$ pregnant women. "'Highly effective” methods included oral contraceptive pill, injection, implant, IUD, tubal ligation, vasectomy, or infertility. "Women could choose more than one method. 'Method data was completely missing for $n=57$ contraceptive users. $n=21$ contraceptive users only selected the "do not know" option. "Dual method use was defined as using condoms to prevent HIV transmission and also using at least one highly effective contraceptive method to prevent pregnancy. 'Long-term methods included IUD, implant, tubal ligation, vasectomy, or infertility.

challenges for family planning education and service delivery in this setting.

Almost all women understood that using condoms is important, and most women reported that they were using condoms to prevent HIV transmission. However, most women overestimated the risk of HIV transmission to their partner when trying to get pregnant, assuming there was a $100 \%$ chance. This type of misconception echoes previous research in South Africa that found many individuals did not believe in serodiscordance or thought that transmission was certain if a condom was not used. ${ }^{8,19}$ Condom-focused prevention messages may also send the stigmatizing message that HIV-affected couples should not have children, as sex without condoms is generally necessary to conceive, ${ }^{29}$ with the exception of self-insemination and safer conception methods that have low availability in resource-limited settings. ${ }^{1}$ Study results emphasize the 


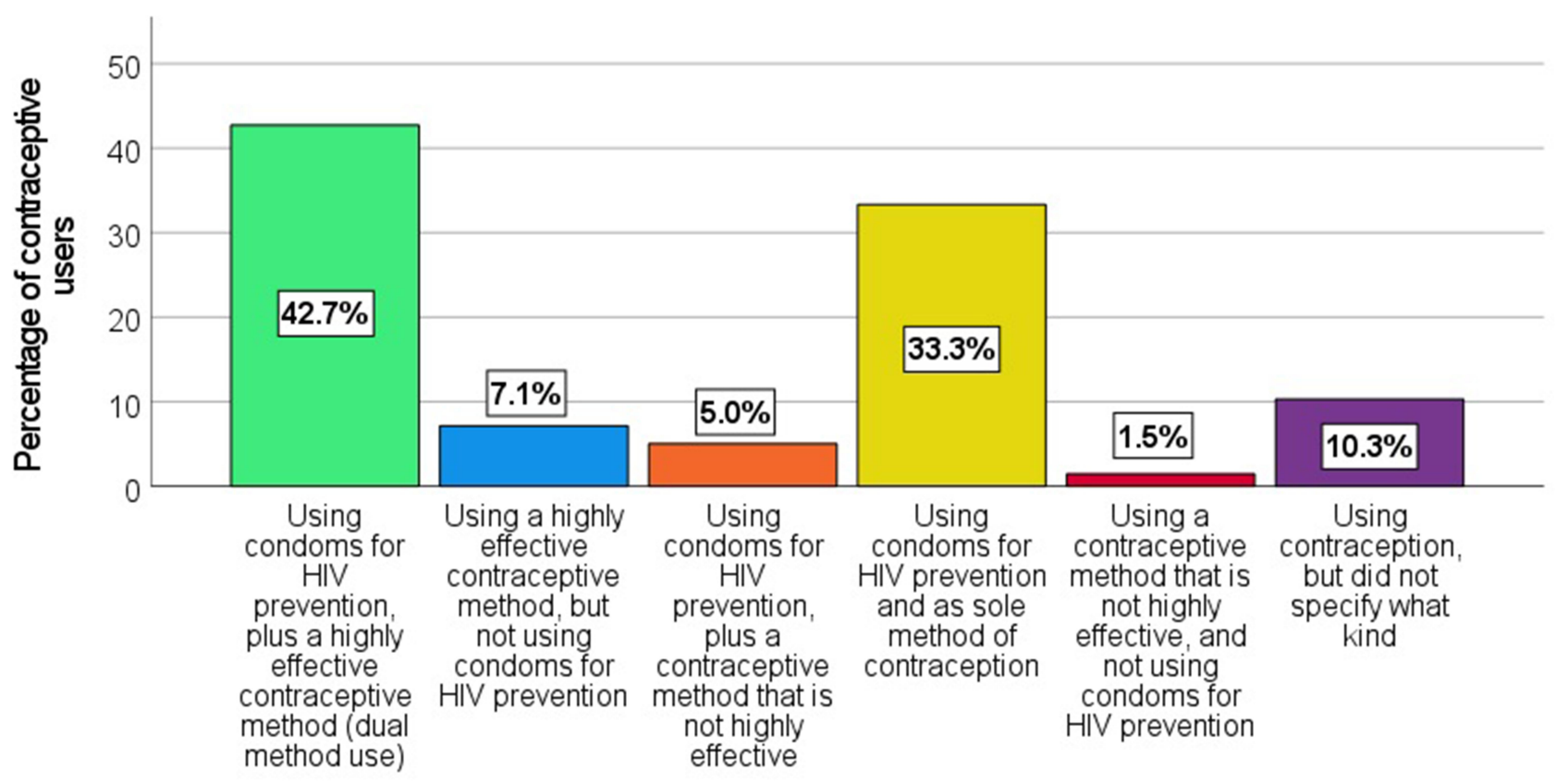

Figure I Breakdown of contraceptive method effectiveness and condom use for HIV prevention among contraceptive users ( $\mathrm{n}=756$ ).

need for health messaging, including information from healthcare workers, ${ }^{30}$ to combat misconceptions, emphasize reproductive freedom, and reduce HIV stigma.

This study found that a majority of women considered the possibility of perinatal transmission and their partner's desires to be important factors in decision-making regarding conception. Previous research has found perinatal HIV transmission to be a frequent concern ${ }^{8,11,12}$ among women for whom marital responsibilities may include childbearing. ${ }^{8,10,12}$ Comprehensive PMTCT programs that include discussion of perinatal transmission may alleviate some of these concerns. Previous research in South Africa also found men's opinions to dominate reproductive decision-making. ${ }^{8} 9$ Although men have a strong influence on decisions to have more children, there are substantial barriers to male partner involvement at antenatal clinics, such as perceiving antenatal clinics as unfriendly toward men. ${ }^{31}$ Strategies to reduce men's discomfort and increase their involvement in periconception fertility planning at clinics may also increase knowledge and uptake of safer conception methods.

Although most women reported using contraception and most reported using condoms for HIV prevention, rates of dual method use were low, in part because many women used condoms for both HIV prevention and pregnancy prevention, without using a highly effective method of contraception for extra protection. Condom failure rates range from $13 \%$ to $21 \%^{28}$ and the $\mathrm{WHO}$ recommends combining it with a highly effective contraceptive method to maximize protection against both pregnancy and HIV. ${ }^{16}$ Dual methods for contraception are recommended until another pregnancy is both desired and safe; among both WHIV and those who are HIV-seronegative, interpregnancy intervals of at least 24-36 months are recommended to optimize maternal and child health outcomes. ${ }^{13}$ Results highlight the limited reach of long-acting reversible contraceptives (LARCs; IUDs and implants) in this population; though used by few women in this sample, these methods are extremely effective, reversible when pregnancy is desired, and last for several years. ${ }^{28}$ These low use rates may reflect barriers to access; although contraception in South Africa is free in the public sector, limited resources, lack of staff training, and supply chain issues may prevent access. ${ }^{32}$ Additionally, healthcare workers' attitudes, HIV stigma, disapproval of premarital sex, and misconceptions about method suitability for young women may also be barriers to access. ${ }^{32}$ Finally, messaging regarding interactions between contraceptive implants and efavirenz-based ART has also created a barrier to implant uptake in South Africa. ${ }^{32}$ The results of this study suggest that additional efforts are needed to reduce barriers to LARC use. Increased emphasis on LARCs in healthcare services and family planning counseling may help more women decide that a LARC is the right choice for them. 


\section{Limitations}

Study limitations associated with generalizability must be considered, eg, loss to follow-up at the 12-month visit. In addition, though this data was cross-sectional, knowledge, attitudes, and practices may change over time. Furthermore, study phase or condition could have potentially influenced some of the results, although the study was not powered to compare these variables by phase or condition after adjusting for cluster effects, longitudinal effects, and multiple comparisons. However, the intervention's primary focus was the PMTCT protocol, and it was not designed to affect all of the variables presented here, so the influence of phase or condition on the data is likely minor. Additionally, although women were required to have a male partner in order to enroll in the study, relationship status was only assessed at baseline, so it is possible that some women may have no longer been in a relationship at the time of the 12-month visit. Also, participants' feelings about family planning, contraceptive methods, information sources, and the nuances of reproductive decision-making were not qualitatively explored. Future research should use mixed methods to examine knowledge, attitudes, and practices more deeply.

\section{Conclusions}

Despite these limitations, this manuscript fills an important gap in recent literature regarding safer conception and family planning knowledge, attitudes, and practices among women with HIV in rural South Africa. Policies and interventions are guided by the population they target, and this study provides an important timely perspective for future efforts regarding contraception, HIV prevention, and childbearing in rural South Africa. Study results present strengths and challenges for safer conception and family planning education and service delivery in the region, with the common goal of helping families to optimize health outcomes and achieve their reproductive goals.

\section{Abbreviations}

ART, antiretroviral therapy; CHC, community health center; IUD, intrauterine device; LARC, long-acting reversible contraceptive; PHIV, people with HIV; PMTCT, prevention of mother-to-child transmission; PYF, Protect Your Family; SCS, safer conception strategies; WHIV, women with HIV; WHO, World Health Organization.

\section{Ethics Approval and Informed Consent}

Prior to study procedures, ethical approval was obtained from the Human Sciences Research Council (HSRC) Research Ethics Committee (protocol approval number REC4/21/08/13), the University of Miami Miller School of Medicine Institutional Review Board (IRB ID: 20130238), and the Mpumalanga provincial government. The study was conducted in accordance with the Declaration of Helsinki. All participants provided written informed consent prior to participation.

\section{Acknowledgments}

The authors would like to thank the Protect Your Family intervention leaders, study staff, and the women participating in this study, without whom this work would not have been possible.

\section{Author Contributions}

All authors made a significant contribution to the work reported, whether that is in the conception, study design, execution, acquisition of data, analysis and interpretation, or in all these areas; took part in drafting, revising or critically reviewing the article; gave final approval of the version to be published; have agreed on the journal to which the article has been submitted; and agree to be accountable for all aspects of the work.

\section{Funding}

This work was primarily funded by the National Institute of Child Health and Human Development (R01HD078187), with additional support from the Miami Center for AIDS Research at the University of Miami Miller School of Medicine, which was funded by the National Institutes of Health (P30AI073961). VJR's work on this manuscript was partially supported by a Ford Foundation Fellowship, administered by the National Academies of Sciences, Engineering, and Medicine.

\section{Disclosure}

The authors have no conflicts of interest.

\section{References}

1. Davies NECG, Ashford G, Bekker L-G, et al. Guidelines to support HIV-affected individuals and couples to achieve pregnancy safely: update 2018. S Afr J Hiv Med. 2018;19(1). doi:10.4102/sajhivmed. v19i1.915. 
2. Heffron R, Pintye J, Matthews LT, Weber S, Mugo N. PrEP as peri-conception HIV prevention for women and men. Curr HIV/ AIDS Rep. 2016;13(3):131-139. doi:10.1007/s11904-016-0312-1

3. Murnane PM, Heffron R, Ronald A, et al. Pre-exposure prophylaxis for HIV-1 prevention does not diminish the pregnancy prevention effectiveness of hormonal contraception. AIDS. 2014;28 (12):1825-1830. doi:10.1097/QAD.0000000000000290

4. Matthews LT, Beyeza-Kashesya J, Cooke I, et al. Consensus statement: supporting safer conception and pregnancy for men and women living with and affected by HIV. AIDS Behav. 2018;22 (6):1713-1724. doi:10.1007/s10461-017-1777-7

5. Davies NE, Matthews LT, Crankshaw TL, Cooper D, Schwartz SR. Supporting HIV prevention and reproductive goals in an HIV-endemic setting: taking safer conception services from policy to practice in South Africa. J Int AIDS Soc. 2017;20(Suppl 1):21271. doi:10.7448/IAS.20.2.21271

6. Department of Health - Republic of South Africa. National contraception and fertility planning policy and service delivery guidelines; 2012. Available from: https://www.health-e.org.za/wp-content /uploads/2014/05/ContraceptionPolicyServiceDelGuidelines2013. pdf. Accessed February 26, 2019.

7. Joseph Davey D, West S, Umutoni V, et al. A systematic review of the current status of safer conception strategies for HIV affected heterosexual couples in sub-Saharan Africa. AIDS Behav. 2018;22 (9):2916-2946. doi:10.1007/s10461-018-2170-x

8. Matthews LT, Crankshaw T, Giddy J, et al. Reproductive decision-making and periconception practices among HIV-positive men and women attending HIV services in Durban, South Africa. AIDS Behav. 2013;17(2):461-470. doi:10.1007/s10461-011-0068-y

9. Cooper D, Harries J, Myer L, Orner P, Bracken H, Zweigenthal V. "Life is still going on": reproductive intentions among HIV-positive women and men in South Africa. Soc Sci Med. 2007;65(10):2186. doi:10.1016/j.socscimed.2007.07.009

10. Willan S, Gibbs A, Petersen I, Jewkes R, Bartels SA. Exploring young women's reproductive decision-making, agency and social norms in South African informal settlements. PLoS One. 2020;15 (4):e0231181. doi:10.1371/journal.pone.0231181

11. van Zyl C, Visser MJ. Reproductive desires of men and women living with HIV: implications for family planning counselling. Reprod Biomed Online. 2015;31(3):434-442. doi:10.1016/j.rbmo.2015.06.004

12. Evens E, Tolley E, Headley J, et al. Identifying factors that influence pregnancy intentions: evidence from South Africa and Malawi. Cult Health Sex. 2015;17(3):374-389. doi:10.1080/13691058.2014.968806

13. World Health Organization. Report of a WHO technical consultation on birth spacing: Geneva, Switzerland 13-15 June 2005. World Health Organization; 2007. Available from: https://apps.who.int/iris/ handle/10665/69855. Accessed November 7, 2019.

14. Yaya S, Uthman OA, Ekholuenetale M, Bishwajit G, Adjiwanou V. Effects of birth spacing on adverse childhood health outcomes: evidence from 34 countries in sub-Saharan Africa. J Matern Fetal Neonatal Med. 2019;1-8.

15. Mugo NR, Heffron R, Donnell D, et al. Increased risk of HIV-1 transmission in pregnancy: a prospective study among African HIV-1-serodiscordant couples. AIDS. 2011;25(15):1887-1895. doi:10.1097/QAD.0b013e32834a9338

16. World Health Organization. Providing Contraceptive Services in the Context of HIV Treatment Programmes. World Health Organization; 2019.

17. Redd AD, Quinn TC, Tobian AA. Frequency and implications of HIV superinfection. Lancet Infect Dis. 2013;13(7):622-628. doi:10.1016/S1473-3099(13)70066-5
18. Matthews LT, Smit JA, Moore L, et al. Periconception HIV risk behavior among men and women reporting HIV-serodiscordant partners in KwaZulu-Natal, South Africa. AIDS Behav. 2015;19 (12):2291-2303. doi:10.1007/s10461-015-1050-x

19. Schwartz SR, West N, Phofa R, et al. Acceptability and preferences for safer conception HIV prevention strategies: a qualitative study. Int $J$ STD AIDS. 2016;27(11):984-992. doi:10.1177/ 0956462415604091

20. Steiner RJ, Black V, Rees H, Schwartz SR, Receipt L. Uptake of safer conception messages in routine HIV care: findings from a prospective cohort of women living with HIV in South Africa. J Acquir Immune Defic Syndr. 2016;72(1):105-113. doi:10.1097/ QAI.0000000000000945

21. Simbayi L, Zuma K, Zungu N, et al. South African National HIV Prevalence, Incidence, Behaviour and Communication Survey, 2017. Human Sciences Research Council; 2019.

22. Rodriguez VJ, LaCabe RP, Privette CK, et al. The Achilles' heel of prevention to mother-to-child transmission of HIV: protocol implementation, uptake, and sustainability. SAHARA J. 2017;14(1):38-52. doi:10.1080/17290376.2017.1375425

23. Peer N, Morojele N, London L. Factors associated with contraceptive use in a rural area in Western Cape Province. S Afr Med J. 2013;103 (6):406-412. doi:10.7196/SAMJ.6201

24. Osuafor GN, Ayiga N Sexual and reproductive decision-making among married and cohabiting women in Mahikeng. 7th African Population Conference; Johannesburg; 2015.

25. Jones D, Peltzer K, Weiss SM, et al. Implementing comprehensive prevention of mother-to-child transmission and HIV prevention for South African couples: study protocol for a randomized controlled trial. Trials. 2014;15. doi:10.1186/1745-6215-15-417

26. Department of Health - Republic of South Africa. National consolidated guidelines for the prevention of mother-to-child transmission of HIV (PMTCT) and the management of HIV in children, adolescents and adults. 2014.

27. Idonije B, Oluba O, Otamere H. A study on knowledge, attitude and practice of contraception among secondary school students in Ekpoma, Nigeria. JPCS. 2011;(2):22-27.

28. Centers for Disease Control and Prevention. Contraception; 2020. Available from: https://www.cdc.gov/reproductivehealth/contracep tion/index.htm. Accessed May 6, 2020.

29. Matthews LT, Moore L, Milford C, et al. "If I don't use a condom ... I would be stressed in my heart that I've done something wrong": routine prevention messages preclude safer conception counseling for HIV-infected men and women in South Africa. AIDS Behav. 2015;19 (9):1666-1675. doi:10.1007/s10461-015-1026-x

30. West N, Schwartz S, Phofa R, et al. "I don't know if this is right ... but this is what I'm offering": healthcare provider knowledge, practice, and attitudes towards safer conception for HIV-affected couples in the context of Southern African guidelines. AIDS Care. 2016;28 (3):390-396. doi:10.1080/09540121.2015.1093596

31. Matseke MG, Ruiter RAC, Barylski N, et al. A qualitative exploration of the meaning and understanding of male partner involvement in pregnancy-related care among men in rural South Africa. $J$ Soc Behav Health Sci. 2017;11.

32. Lince-Deroche N, Pleaner M, Morroni C, et al. Achieving universal access to sexual and reproductive health services: the potential and pitfalls for contraceptive services in South Africa. $S$ Afr Health Rev. 2016;2016(1):95-108. 
Open Access Journal of Contraception

Dovepress

\section{Publish your work in this journal}

Open Access Journal of Contraception is an international, peerreviewed, open access, online journal, publishing original research, reports, reviews and commentaries on all areas of contraception. In addition to clinical research, demographics and health-related aspects, the journal welcomes new findings in animal and preclinica studies relating to understanding the biological mechanisms and practical development of new contraceptive agents. The manuscript management system is completely online and includes a very quick and fair peer-review system. Visit http://www.dovepress.com/testimonials. php to read real quotes from published authors. 